\title{
Energy Maps for Long-Range Path Planning for Small- and Micro - UAVs
}

\author{
Anjan Chakrabarty* and Jack W. Langelaan ${ }^{\dagger}$ \\ The Pennsylvania State University, University Park, PA, 16802, USA
}

\begin{abstract}
This paper presents a method for planning trajectories which exploit atmospheric energy to enable long distance, long duration flights by small and micro unmanned aerial vehicles. It introduces the energy map, which computes the minimum total energy required to reach the goal from an arbitrary starting point while accounting for the effect of arbitrary wind fields. The energy map provides the path to the goal as a sequence of way points, the optimal speeds to fly for each segment between way points and the heading required to fly along a segment. Since the energy map is based on the minimum total energy required to reach the goal it immediately answers the question of existence of a feasible solution for a particular starting point and initial total energy. The energy map can also be used in conjunction with other components of a cost function (e.g. time to reach the goal) for use in generic trajectory planners.
\end{abstract}

\section{Introduction}

SMALl AND Micro UAVs face severe limits on the fuel that can be carried, greatly reducing both endurance Nand range. In addition, the best L/D attainable for small and micro UAVs is typically much smaller than for larger aircraft because of the smaller Reynolds numbers. This further reduces performance. Thus a major limitation in developing practical mini and micro UAVs is the energy required for long range, long endurance operations.

Battery technology is continually improving. Clearly improvements in battery technology will result in extended range and endurance. However, immediate performance gains are possible by harvesting energy from the atmosphere through soaring flight. Large birds such as eagles, hawks and condors as well as human sailplane and hang glider pilots routinely use soaring flight to remain aloft for many hours and traverse hundreds of kilometers without flapping wings or the use of engines.

The most common means of atmospheric energy harvesting exploits vertical air motion (i.e. updrafts). Updrafts have three main causes: uneven heating of the ground, which produces buoyant instabilities known as thermals; long period oscillations of the atmosphere, generally called wave, which occurs in the lee of large mountain ranges; and orographic lift, where wind is deflected by the slopes of hills and mountains. Typically updrafts have lifespans measured in minutes (for thermals) to hours or days (for ridge and wave lift). Ridge lift and wave are predictable phenomena, and thus one can use trajectory planning techniques to compute paths which exploit vertical air motion to enable extremely long distance or duration flights.

The focus of this paper is on planning long-distance soaring trajectories which harvest energy available from a known wind field (this may be obtained from predictions generated using meteorological forecasting tools such as $M 5^{1}$ ). Previous research addressed this problem using a probabilistic road map approach ${ }^{2}$ and using a gradient-based optimization. ${ }^{3}$ In this paper we define a total energy map, which computes the total energy required to reach the goal from any starting point in the environment. This total energy map is computed by first defining nodes (or way points) distributed throughout the environment. Using wavefront expansion from the goal we compute the speed to fly which minimizes energy loss for flight between two nodes, and compute the minimum total energy required to reach the goal from a given node.

\footnotetext{
* Graduate Student, Department of Aerospace Engineering, The Pennsylvania State University.

$\dagger$ Assistant Professor, Department of Aerospace Engineering, The Pennsylvania State University, Senior Member AIAA

Copyright (C) 2009 by Anjan Chakrabarty and Jack W. Langelaan. Published by the American Institute of Aeronautics and Astronautics, Inc. with permission.
} 
The remainder of this paper is organized as follows. Section II discusses previous relevant works in this field. Section III describes the discretization of the environment and the calculation of energy map. Section IV discusses the dynamics and kinematics of flight and describes the total energy of the system and defines the energy minimization problem. Section V presents results of some sample problems. Section VIdescribes the long range planning in presence of complicated wind fields and finally Section VII presents concluding remarks.

\section{Previous and Related Work}

AICH AND VARIED literature exists in the field of optimal static soaring trajectories with the application A of human-piloted soaring flight. Various aspects of optimal static soaring have been addressed, including the optimal speed to fly between thermals of known strength (the MacCready problem, ${ }^{4,5}$ the final glide problem, ${ }^{6}$ and "dolphin" flight along regions of alternating lift and sink ${ }^{7-9}$ de Jong ${ }^{10}$ describes a geometric approach to trajectory optimization and also discusses the optimal deviation from course to minimize time to a goal in a given lift field. Much of this research is directly applicable to the problem of trajectory generation for autonomous soaring flight, but it assumes limited types of known lift distributions (e.g. sinusoidally varying lift ${ }^{11}$ or "square wave" lift $^{12}$ ) and generally do not consider the effects of horizontal wind components.

Autonomous static soaring is now becoming the focus of more research. Simulation results of thermal flight are reported by Allen $(2005)^{13}$ and flight test results are presented in Allen (2007). ${ }^{14}$ Edwards reports very impressive results of autonomous thermal soaring. ${ }^{15}$ However, these do not consider the problem of trajectory planning.

Wind routing for powered aircraft has been considered for both crewed and uncrewed aircraft. Rubio describes a planning method based on genetic algorithms; ${ }^{16}$ Jardin discusses a method based on neighborhood optimal control. ${ }^{17}$ Neither of these approaches consider the possibility of harvesting energy from vertical components of the wind field.

\section{The Energy Map}

Cellular decomposition approaches to robot path planning have been used very successfully. The robot's configuration space is divided into a finite number of regions, and the planning problem is reduced to finding a sequence of neighboring cells between the start and goal region (e.g. Stentz ${ }^{18}$ ). These graphbased techniques have been used very successfully in many wheeled ground robot path planning problems and have been used for some UAV planning problems, typically radar evasion. ${ }^{19}$

However, these techniques typically only consider a fixed cost for a transition between nodes in a graph (e.g. time required) and vehicle speed is kept constant. In aircraft applications total energy can be a critical parameter in trajectory planning (for example, when considering the fuel required to reach the goal). Both environmental and control parameters can affect the energy required for a particular transition: a head wind will increase the required total energy, as will flying at non-optimal airspeed. Thus any graph-based planning technique will require a means of accounting for environmental and control conditions in the analysis of costs of transitions between nodes or cells.

One approach is to compute the minimum total energy required to complete each of the transitions in the graph (note that the energy $e_{i j}$ required to complete a transition from node $i$ to node $j$ is not necessarily the same as $e_{j i}$ ). The trajectory planning problem then becomes a problem of finding the minimum energy path through the digraph to the goal, i.e. the sequence of nodes $\mathbf{n}$ which minimizes the energy required to reach the goal

$$
e_{\text {goal }}=\sum_{i \in \mathbf{n}, j \in \mathbf{n}} e_{i j}
$$

Several techniques have been developed to compute cost-minimizing paths through a graph. Dijkstra's Algorithm is not applicable here because it is restricted to problems where edge costs are non-negative, and the Bellman-Ford algorithm encounters problems when negative cycles exist. ${ }^{20}$ In this application a nonnegative edge cost implies energy gain, which occurs with flight through an updraft of sufficient strength. Negative energy cycles occur when the aircraft flies repeatedly between two nodes, gaining energy with each trip. 
This paper introduces the concept of an energy map, which defines a bound on the minimum energy required to reach the goal from anywhere in the environment. The solution is based on wavefront expansion.

\section{A. Map Definition and Minimum Energy Paths}

To define the energy map, the environment is first seeded with waypoints (or nodes) and edges. This set of nodes $i=\{i=0 \ldots m\}$ (with $i=0$ denoting the goal) and edges $i j=\{i=0 \ldots m, j=0 \ldots m\}$ connecting nodes define the allowable paths to the goal. Each edge $i j$ is assigned a constant wind vector $\mathbf{w}_{i j}$ with components $w_{x, i j}, w_{y, i j}$, and $w_{z, i j}$. Wind field information is assumed to be available a priori.

After the set of nodes and the set of allowable transitions has been defined, the cost of each transition is computed. In steady state flight, the energy $e_{i j}$ required to fly from node $i$ to $j$ is a function of the wind vector $\mathbf{w}_{i j}$, air speed $v_{a}$ and throttle setting $T$. The heading $\psi_{i j}$ required to fly along the desired ground track between the two nodes is a function of the horizontal component of the wind field and the air speed. The problem of computing $v_{a}$ which minimizes the energy required for transition $i j$ is discussed in Section IV.

It is assumed that the vehicle is in a trimmed, steady state condition during each transition over an edge $i j$, and the time required to change from one trim condition to the next as a node is passed is short compared with the length of time required to complete a transition.

Aside from the constraint that the time required to complete a transition at a particular trim condition is long compared with the time required to change trim conditions from one transition to the next, node placement is arbitrary. Higher node density can thus be used in regions where spatial gradients in wind field are large, allowing higher resolution trajectories when necessary.

\section{B. Minimum Energy Paths via Wavefront Expansion}

One approach to solving the problem of minimum energy to the goal is via wavefront expansion. The set of nodes is ordered by increasing distance to goal, and transitions which simultaneously satisfy the condition of being to a neighboring node and reducing the distance to the goal are defined as allowable.

The energy to reach the goal is computed for the group of nodes nearest the goal, and the energy corresponding to each node is defined as their respective costs-to-go. For the next group of nodes the energy required to reach nearest neighbors in the first group is computed, and the cost-to-go for each node is the minimum total energy over all possible paths to the goal. The process continues until energy to goal has been computed for each node. This is a breadth-first dynamic programming approach, and with the constraint that transitions must always end in nodes nearer to the goal, the resulting energy map gives an upper bound on the minimum energy required to reach the goal from any point in the environment. An example grid is shown in Figure 1.

In this approach each node is encoded with the total energy required to reach the goal (i.e. the cost

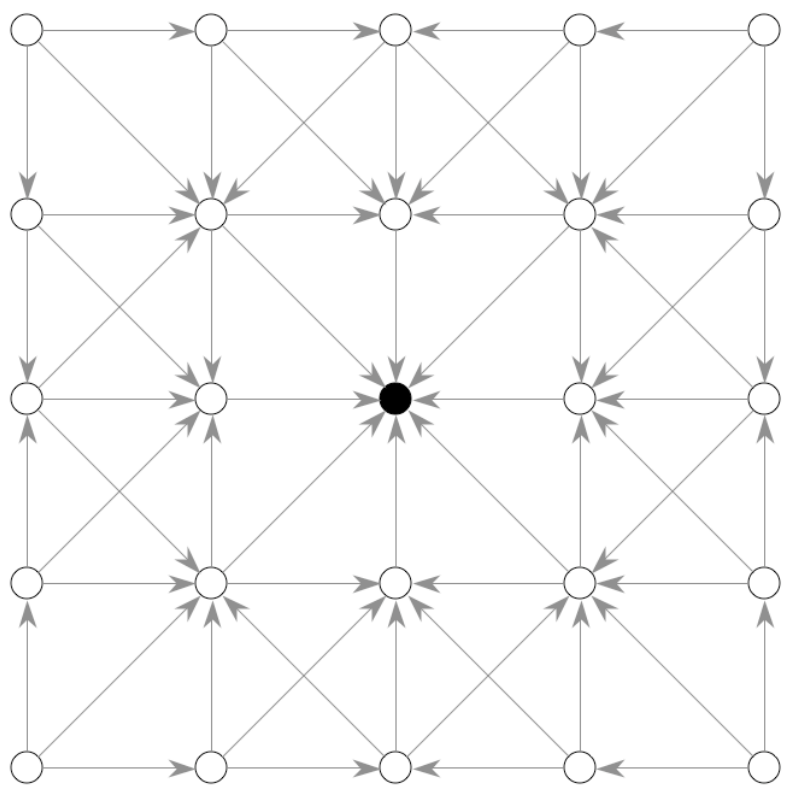

Figure 1. Sample regular Cartesian grid showing allowable transitions for wavefront expansion. The goal is at the center of the grid to go), the next node in the path to the goal, and the control inputs (speed to fly and heading) required to reach the next node. The energy map thus encodes the complete path to the goal from anywhere in the environment. Further, it provides a means to check the feasibility of a path to the goal for an aircraft with a particular initial position and initial total energy.

Note that paths found using this method are not necessarily minimum energy approaches: since paths are constrained to always approach the goal, trajectories which are more energy efficient may exist. Such a path would first proceed away from the goal before turning towards it. However, relaxing the constraint that 
the goal must be approached would make the wave front expansion approach computationally intractable for large environments.

A critical criterion in hardware implementation is the time required to compute a solution. Fast planning solutions permit on-line re-planning as changes in the environment (i.e. the wind field) occur. Here, the definition of nodes and the allowable transitions between nodes is pre-determined and stored in a tree. Computing the costs of allowable transitions and maintaining the minimum cost to go for each node can thus be performed quickly.

\section{Vehicle Kinematics and Energetics}

T IS ASSUMED that an on-board controller is able to follow heading, airspeed and throttle commands. Moreover, it is assumed that response to step changes in commands is very fast compared with the duration of a particular command. Hence a point mass model is sufficient to describe vehicle motion for planning purposes (Figure 2). Vehicle kinematics are given by

$$
\begin{aligned}
\dot{x} & =v_{a} \cos \gamma \cos \psi+w_{x} \\
\dot{y} & =v_{a} \cos \gamma \sin \psi+w_{y} \\
\dot{z} & =v_{a} \sin \gamma+w_{z}
\end{aligned}
$$

where $v_{a}$ is airspeed, $\psi$ is heading and $w_{x}, w_{y}$ and $w_{z}$ are the three components of the $3 \mathrm{D}$ wind vector.

The glide path angle $\gamma$ is a function of airspeed $v_{a}$ and throttle setting $T$, and can be obtained for steady flight. From (Figure 3) resolving forces parallel and perpendicular to the flight path,

$$
\begin{aligned}
& m g \cos \gamma=L+T \sin \alpha_{i} \\
& m g \sin \gamma=D-T \cos \alpha_{i}
\end{aligned}
$$

where $m$ is mass of the vehicle and $\alpha_{i}$ is the angle between the thrust axis and the flight path. (NB if the thrust axis is aligned with the aircraft's body $x$ axis then $\alpha_{i}=\alpha$ )

It is assumed that the flight path angle $\gamma$ is small, hence $\sin \gamma \approx \gamma$ and $\cos \gamma \approx 1$. A further simplifying assumption (somewhat less accurate) is that thrust is always aligned with the flight path angle (i.e. $\alpha_{i}$ is zero). From Equation 5

$$
m g=L=\frac{1}{2} \rho v_{a}^{2} S C_{L}
$$

therefore

$$
C_{L}=\frac{2 m g}{\rho v_{a}^{2} S}
$$

Here $C_{L}$ is lift coefficient, $\rho$ is density of the air, and $S$ is wing area. A polynomial approximation is used for the aircraft's drag polar:

$$
C_{D}=\sum_{i=0}^{n} a_{i} C_{L}^{i}
$$

Thus the drag force is

$$
D=\frac{1}{2} \rho v_{a}^{2} S \sum_{i=0}^{n} a_{i} C_{L}^{i}
$$

Typically a second order polynomial is used to represent drag coefficient. However, this is often only valid over a fairly narrow speed range, and here a fourth order polynomial is used. 
Substituting into Equation 6, the flight path angle for a particular speed and thrust can thus be computed as

$$
m g \gamma=\frac{1}{2} \rho v_{a}^{2} S \sum_{i=0}^{n} a_{i} C_{L}^{i}-T
$$

The vehicle's flight path is thus completely specified by inputs $\mathbf{u}=\left[v_{a} \psi T\right]^{T}$ and wind speed $\mathbf{w}$. This model is adequate as long as the length of time of each trajectory segment is large compared with the time constant of the vehicle's step response with respect to the inputs $\mathbf{u}$. For the remainder of this paper we consider only gliding flight, hence $T=0$.

\section{A. Flight between two nodes}

Here the kinematics to fly between two nodes is defined. The line segment joining two successive nodes is the desired ground track. We decompose the velocity of the vehicle in in-track $v_{t}$ and cross track $v_{c}$ components. Clearly to maintain flight along the desired ground track $v_{c}=0$. We also decompose the wind vector in in-track $w_{t}$ and cross-track $w_{c}$ components. $v_{g}$ is the ground speed of the vehicle (Figure 3).

From Figure 3 we can obtain the relationship between air speed, ground speed, heading and ground track for an arbitrary horizontal component of wind:

$$
\begin{aligned}
v_{t} & =\sqrt{v_{a}^{2} \cos ^{2} \gamma-w_{c}^{2}} \\
v_{g} & =v_{t}+w_{t} \\
v_{a} \cos \gamma \sin \beta & =w_{c}
\end{aligned}
$$

where $v_{g}$ is the magnitude of the ground speed, $v_{a} \cos \gamma$ is the projection of the airspeed vector onto the horizontal plane and $\beta$ is the angle between the airspeed vector and the desired ground track. Typically the flight path angle $\gamma$ is small, and we shall assume that $\cos \gamma=1$.

The aircraft heading to maintain the desired ground track is $\psi=\psi_{t}-\beta$. Hence

$$
\psi=\psi_{t}-\sin ^{-1} \frac{w_{c}}{v_{a}}
$$

Clearly heading $\psi$ is dependent on airspeed $v_{a}$. The problem now is to determine the optimal value of airspeed $v_{a}$ for flight between two nodes.

\section{B. Total Energy Maximization}

We shall determine the steady state airspeed which minimizes the energy lost over a segment (or equivalently, maximizes the energy gained). Ignoring stored energy (i.e. fuel or electrical energy stored in batteries), total energy is

$$
E=m g h+\frac{m}{2}\left(\dot{x}_{i}^{2}+\dot{y}_{i}^{2}+\dot{z}_{i}^{2}\right)
$$

where $h$ is altitude. We define specific total energy as

$$
e=\frac{E}{m g}=h+\frac{1}{2 g}\left(\dot{x}_{i}^{2}+\dot{y}_{i}^{2}+\dot{z}_{i}^{2}\right)
$$

For steady state flight, maximizing energy gained over a segment is equivalent to maximizing $\frac{\dot{e}}{v_{g}}$. In zero wind for gliding flight this will result in flight at best $L / D$. The rate of change of specific energy is

$$
\dot{e}=\dot{h}+\frac{1}{g}(\dot{x} \ddot{x}+\dot{y} \ddot{y}+\dot{z} \ddot{z})
$$


In steady flight acceleration is zero, hence

$$
\dot{e}=\dot{h}=-\dot{z}
$$

Recall that $z$ is positive down. Combining Equations 4, 12 and 13,

$$
\frac{\dot{e}}{v_{g}}=\frac{-\left(v_{a} \sin \gamma+w_{z}\right)}{\sqrt{v_{a}^{2} \cos ^{2} \gamma-w_{c}^{2}}+w_{t}}
$$

Recall that we assume that the flight path angle $\gamma$ is small, hence

$$
\frac{\dot{e}}{v_{g}}=\frac{-\left(v_{a} \gamma+w_{z}\right)}{\sqrt{v_{a}^{2}-w_{c}^{2}}+w_{t}}
$$

Since $e$ has dimension distance, $\frac{\dot{e}}{v_{g}}$ is a dimensionless quantity. Equation 11 shows that for gliding flight (i.e. zero thrust) flight path angle is a function only of airspeed (because in trimmed flight $C_{L}$ is a function of airspeed). Thus we can compute the airspeed which maximizes energy gained over a segment. Note that in zero wind the energy change is always negative. ${ }^{a}$

The cost of a transition is defined as

$$
c_{i j}=-\frac{\dot{e}}{v_{g}}
$$

Thus energy loss is a positive quantity and energy gain is a negative quantity. We use MatLab's bounded minimization function fminbnd to find $v_{a}^{\text {opt }}$ which minimizes $c_{i j}$ while ensuring that airspeed limits (stall and maximum speed) are not exceeded. Once $v_{a}^{o p t}$ has been computed the required heading to maintain the desired ground track between the start and end nodes is computed from Equation 15.

Thus we have a means to compute the optimal speed to fly and heading for flight between two nodes under arbitrary wind. This is done for each of the allowable transitions in the digraph, and the technique described earlier is used to compute the minimum energy path to the goal.

\section{Energy Maps for Sample Wind Fields}

$\mathrm{T}_{\mathrm{c}}^{\mathrm{s}}$ O DEMONSTRATE THE energy mapping approach we compute energy maps for some simple wind fields. Calculations were performed for an RnR Products SB-XC glider; parameters are given in Table 1. This will provide some intuitive understanding of the energy maps which are produced.

\section{A. Horizontal air motion}

We first compare the zero wind case with uniform wind blowing along the $x$ axis (Figure 4). The total energy required to reach the goal is shown as a mesh surface, and flight paths to the goal are shown as black streamlines on the plane $z=0$.

For the zero wind case we obtain the expected result: the energy required to reach the goal increases linearly with distance to goal. The stream lines in the graph show the path the vehicle should follow to satisfy the requirement for the minimum energy. Adding a horizontal wind component "tilts" the cone, so that starting points downwind of the goal require more initial total energy to reach the goal than starting points upwind of the goal. The path to the goal also modifies accordingly. This also matches intuition.

Note that uniform Cartesian grids are used, and the effect of the grid can be seen in the solutions. A polar grid will produce a smooth cone in the zero wind case.

The second case involves a non-uniform wind field consisting of constant wind shear. Here the horizontal component of wind varies linearly from $w_{x}=-10 \mathrm{~m} / \mathrm{s}$ at $y=-5000$ to $w_{x}=10 \mathrm{~m} / \mathrm{s}$ at $y=5000$. The energy map and paths to goal are shown in Figure 5.

Matching intuition, the surface defining the energy map is now "twisted". Optimal flight paths show the vehicle maximizing exposure to favorable winds (or minimizing exposure to unfavorable wind): for starting points in the regions $x>0, y>0$ and $x<0, y<0$ flight paths remain in the region of high tailwind before turning to approach the goal. For starting points in the regions $x>0, y<0$ and $x>0, y<0$ the same is true: flight paths begin with motion towards regions of more favorable wind before turning towards the goal.

${ }^{\text {a }}$ For powered aircraft the change in total energy should include fuel burned, so the change in total energy will also be negative once this is taken into account. 


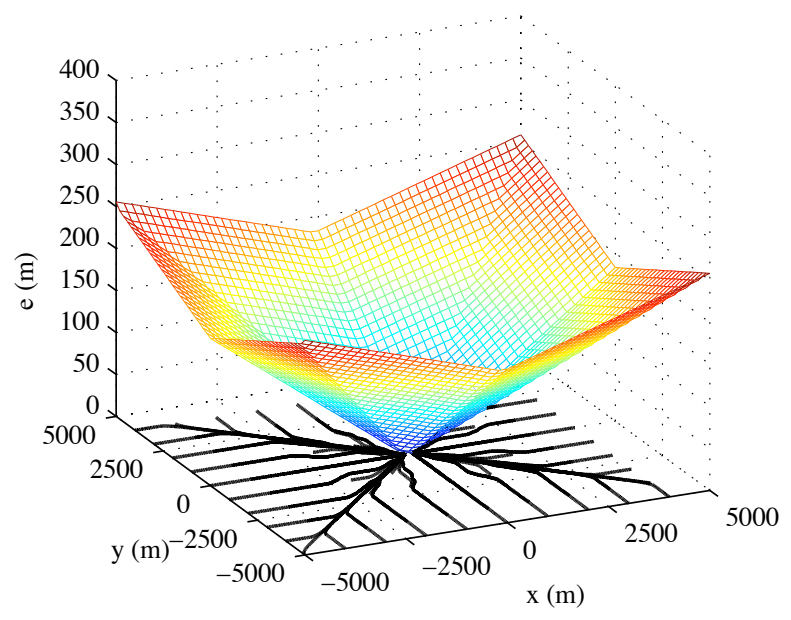

(a) Zero wind

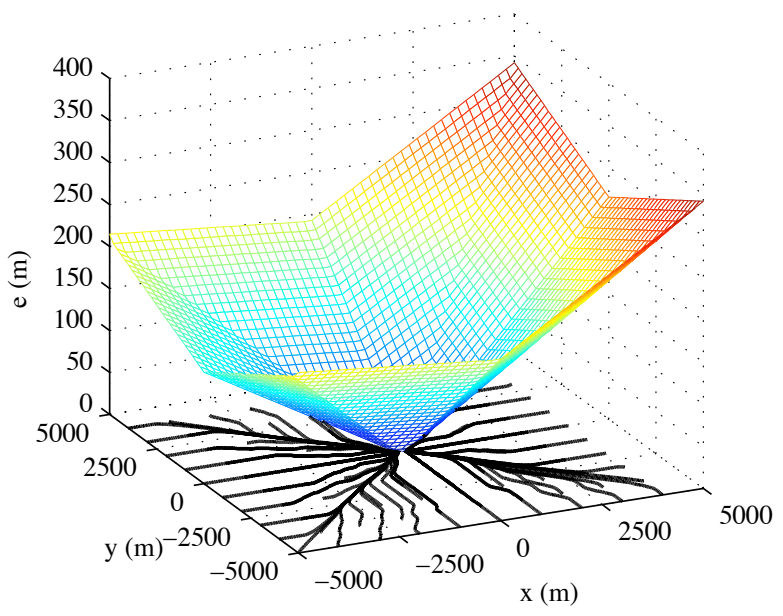

(b) $5 \mathrm{~m} / \mathrm{s}$ wind in the positive $\mathrm{x}$ direction

Figure 4. Energy maps for a uniform horizontal wind fields.

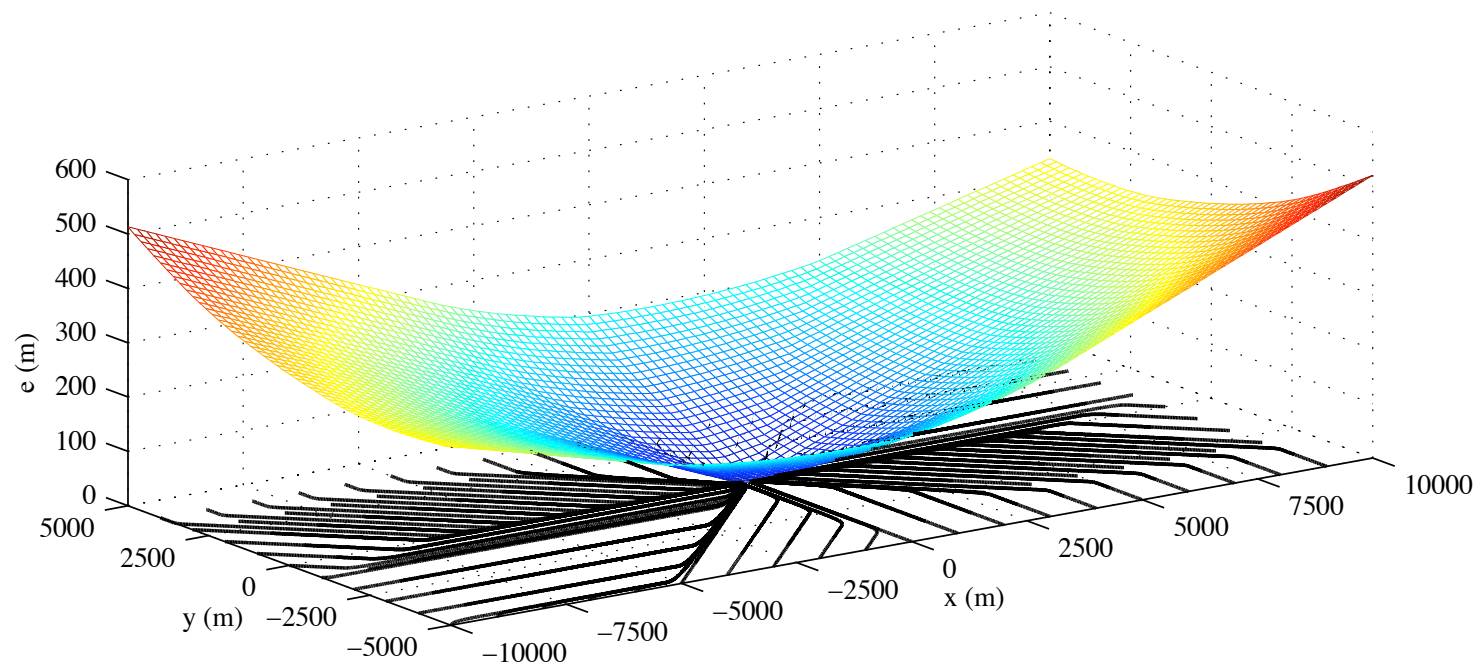

Figure 5. Constant wind shear energy map and minimum energy flight paths. 


\section{B. Vertical air motion}

In contrast to horizontal air motion, vertical air motion allows energy gain during flight. In principle this means that a vehicle can reach a goal with negative initial energy: this may occur in cases where the goal is at higher altitude than the starting position (and thus the vehicle begins with negative potential energy), or when the goal must be reached with a large amount of kinetic energy.

For these simulations, we constrain the starting energy to be non-negative. This will result in regions which show zero initial energy required: this indicates the presence of a region of upward air motion sufficient to permit climbing flight (i.e. gain of total energy). The non-negative initial total energy is effectively a constraint on the starting altitude: since kinetic energy cannot be negative, positive total energy corresponds to a requirement of positive height above a datum (e.g. terrain).

To illustrate the effect of upwards air motion we introduce a simple example. Consider a uniform Cartesian grid of $10 \times 10$ nodes. A region of upwards moving air exists along the nodes defined by $x=5$ and $x=6$. Figure 6a shows the energy map for this wind field. Several distinct regions are visible in the energy map.

There is a region near the goal where the most favorable strategy is flight straight towards the goal. The size of the "direct to goal" region will vary depending on the strength of upwards moving air.

There is a significant region which shows zero initial energy required: this arises from the non-negative starting energy constraint. This constraint is conservative: while the energy map shows that motion with zero energy change is possible, in fact the vehicle may be able to gain significant amounts of energy here. Thus it will reach the goal with more than zero energy remaining (i.e. an excess of altitude or speed).

Outside of the direct to goal region the most energetically favorable flight paths take the glider to the region of rising air and then to the goal. As we shall see later, the size of the zone of attraction of the regions of rising air depends on the magnitude of the upwards air motion (and thus on the rate of energy gain in this region).

Figure $6 \mathrm{~b}$ shows a vector plot of paths to goal and highlights the path to goal from $(10,10)$. The direct to goal region is clear, as are the paths to the region where energy can be gained. The highlighted path shows the vehicle staying in the energy gain region as long as possible (while still satisfying the constraint of continuous motion towards the goal)

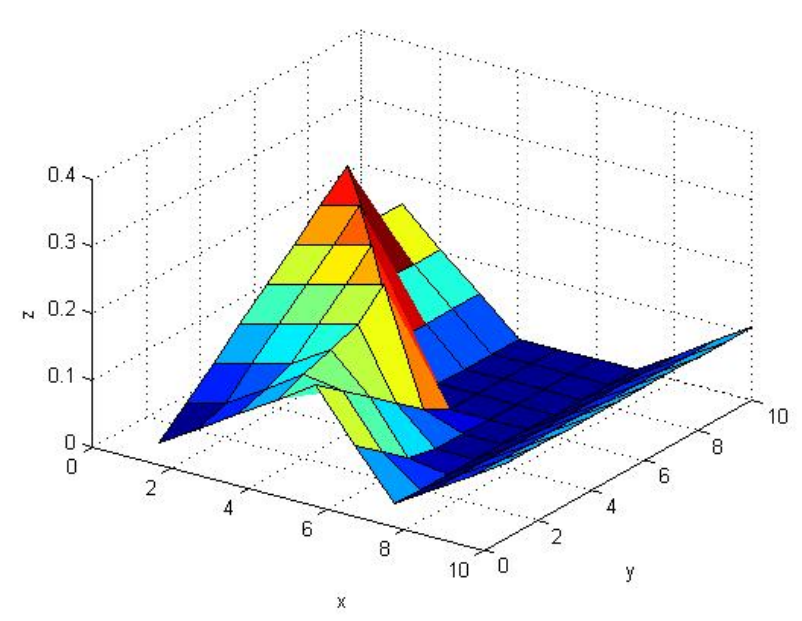

(a) Energy map (z axis is total energy)

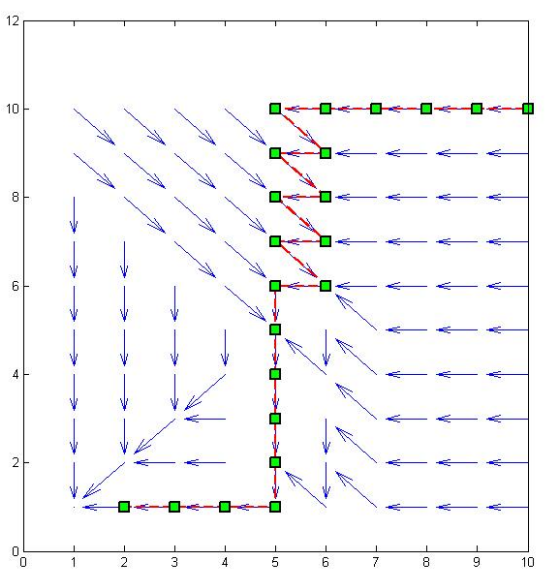

(b) Path to goal

Figure 6. Energy map and paths to goal for an environment with a region of upwards air motion.

\section{Long Range Path Planning}

Ne PURPose of the energy map is to determine the feasibility of flight from any location in the environment to a goal. The second is to determine feasible paths to the goal in real time, thus computation time is a critical consideration.

Here we consider the problem of flight to a distant goal in terrain representative of the ridges of the 


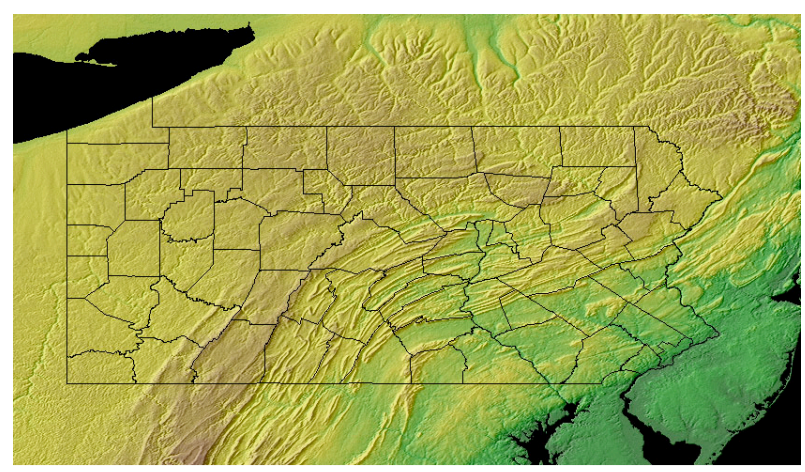

(a) Digital elevation map of Pennsylvania

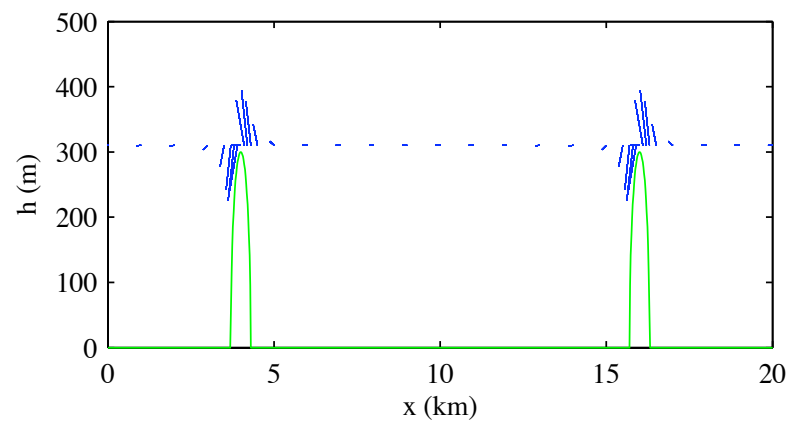

(b) Cross section of parallel ridges and wind field

Figure 7. Left: Topography of central Pennsylvania; Right: Schematic of ridges and potential flow solution of wind field.

Appalachian Mountains of central Pennsylvania (Figure 7). Two parallel ridges are separated by $12 \mathrm{~km}$. A global coordinate frame is defined with $y$ parallel to the ridges, so that the ridge centerlines are located at $x=4 \mathrm{~km}$ and $x=16 \mathrm{~km}$. Each ridge is modeled as an infinitely long hemi-cylinder with radius $300 \mathrm{~m}$, and potential flow is used to compute the wind field. Note that potential flow cannot model flow separation on the downwind side of the ridges, but it is along the upwind side that one finds upwards (and thus favorable) air motion. Intuition suggests (and results will show) that the glider will tend to follow the upwind sides of ridges, thus the flow on the downwind sides of ridges is less critical to trajectory planning (except for the times when the vehicle must traverse these non-favorable regions).

The origin is located at $(0,0)$ and the energy map is computed for an area defined by $0 \leq x \leq 20 \mathrm{~km}$ and $0 \leq y \leq 100 \mathrm{~km}$. Here we compute the wind field at an altitude of $310 \mathrm{~m}$ and it is assumed that the wind field does not vary with altitude. Clearly this assumption is not true for potential flow over a hemi-cylindrical ridge, but it allows the use of graph-based planning without excessive graph size.

Recall that the method discussed here does not required uniform grid spacing, and in some cases uniform grid spacing will either result in inaccurate energy maps (because it is assumed that the wind field is constant over an edge) or excessive computational requirements. For this example a non-uniform Cartesian grid is used, with finer grid spacing over the ridges (where the wind field changes rapidly) and wider spacing between the ridges (where the wind field is roughly constant). Figure $7 \mathrm{~b}$ shows a vector plot of the computed wind field at the $x$ coordinates of the grid. Spacing varies from a minimum of $100 \mathrm{~m}$ to a maximum of $1000 \mathrm{~m}$. Grid spacing in the $y$ direction is constant at $1000 \mathrm{~m}$.

Energy maps for two different magnitudes of wind field are shown in Figure 8: the top figure shows the energy map and paths to fly for a wind field resulting from $w_{x, \infty}=-5 \mathrm{~m} / \mathrm{s}$ (which results in maximum vertical component of wind of approximately $3 \mathrm{~m} / \mathrm{s}$ along the ridge), the bottom figure shows the energy map and paths to fly for $w_{x, \infty}=-1 \mathrm{~m} / \mathrm{s}$ (which results in maximum vertical component of wind of approximately $0.6 \mathrm{~m} / \mathrm{s}$ along the ridge). The minimum sink rate for the vehicle used in these examples (i.e. the minimum rate of altitude loss in still air) is $0.56 \mathrm{~m} / \mathrm{s}$, hence the maximum vertical wind speed in the second example is only slightly larger than the minimum possible to enable energy gain.

Figure 8a shows the energy plot for the high wind case. Notice the nearly constant "troughs" of starting energy along the ridges, indicating that energy can be gained very quickly near the ridges. If the glider starts in the region of upwards moving air, only enough energy to begin stable flight is necessary to permit a flight to the goal, even if the goal is more than $100 \mathrm{~km}$ distant. The direct to goal zone is therefore quite small: because so much energy is available at the ridges, it is advantageous to fly towards the ridge before proceeding to the goal.

The "far" ridge has a significant region of attraction: once the starting position is in the region defined by $y_{0} \geq 27 \mathrm{~km}$ and $x_{0} \geq 11 \mathrm{~km}$, flight paths take the aircraft first to the far ridge, then along the ridge in the $-y$ direction, and finally to the goal along the line of nodes defined by $y=0$. If $x_{0}<11 \mathrm{~km}$ or $y_{0}<27 \mathrm{~km}$ and $x_{0}<16 \mathrm{~km}$ then flight paths go to the "near" ridge before proceeding to the goal along $y=0$.

As expected, the slope of the energy map is significantly steeper for upwind transitions than for downwind transitions. Significantly more energy required to travel a given distance with a headwind than with a tailwind. 


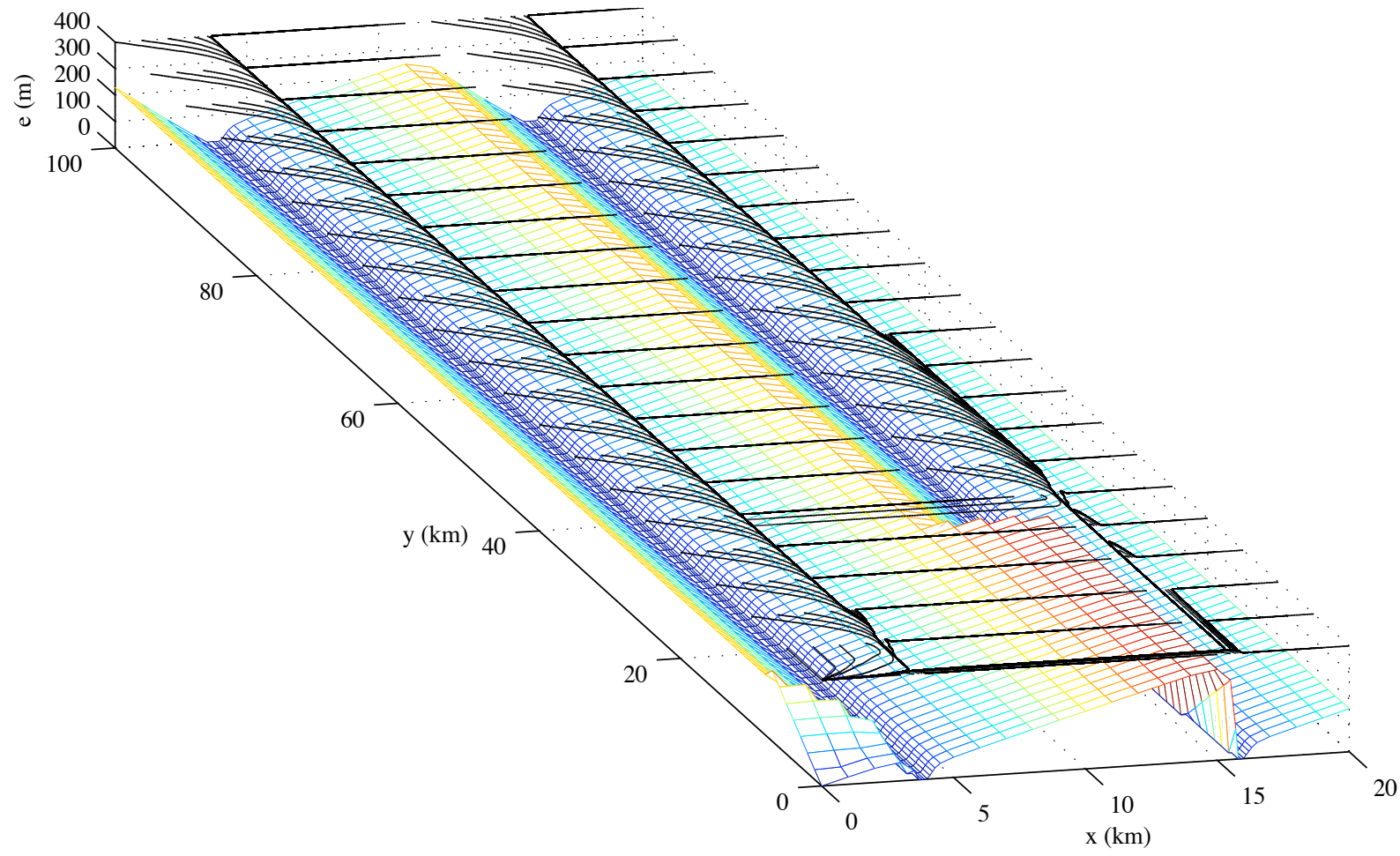

(a) $w_{x, \infty}=-5$

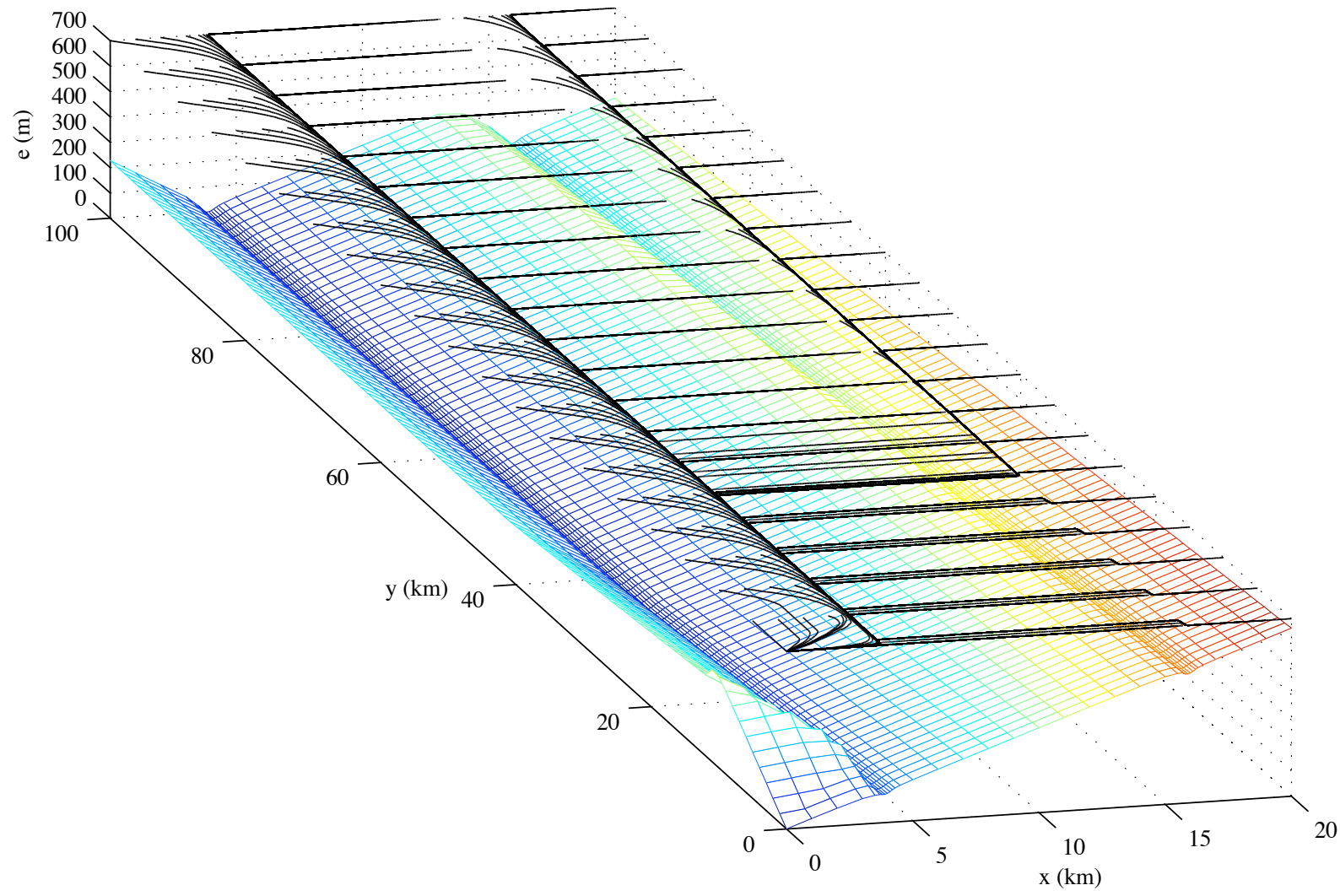

(b) $w_{x, \infty}=-1$

Figure 8. Energy maps (gridded surface) and minimum energy flight paths (stream lines) for parallel ridges. 
Figure 8b shows the energy map for the low wind case. Qualitatively the energy map is similar to the high wind case, but because less energy is available from the environment there are differences.

First, the direct to goal region is larger, reaching $10 \mathrm{~km}$ in the $y$ direction versus $6 \mathrm{~km}$ in the $y$ direction for the high wind case. Second, the troughs of starting energy are no as deep, indicating that even if a glider starts in a region of upwards moving air significant starting energy may be required to reach the goal. Third, the region of attraction of the "far" ridge is smaller. In fact, for starting position with $y_{0}<25 \mathrm{~km}$ the far ridge is essentially ignored. This is in part caused by the path constraint, but it is interesting to note the difference from the high wind case. The maximum required initial specific total energy for the low wind case is almost $700 \mathrm{~m}$, compared with approximately $350 \mathrm{~m}$ for the high wind case.

\section{A. Computation time}

One method of handling uncertainty in the wind field is to re-plan trajectories as updated wind information is obtained. This requires real-time trajectory planning. For planning purposes, a trajectory computation is "real-time" if the time required to plan a path is less than the time required to fly one segment of the trajectory. The energy maps above consist of 3700 nodes (37 in the $x$ direction, 100 in the $y$ direction). Generating the energy maps using MatLab on a 2.67GHz Dell laptop took 19 seconds. For grids of 10000 nodes, energy map generation took approximately 55 seconds. For the SB-XC, best L/D occurs at approximately $16 \mathrm{~m} / \mathrm{s}$. Node spacing of $300 \mathrm{~m}$ to $1000 \mathrm{~m}$ will thus allow trajectory computation in less time than required to fly a single segment.

In the implementation described here, the set of nodes and the set of allowable transitions is pre-computed, requiring only the wavefront expansion and computation of optimal airspeeds to be done in real time. MatLab's fminbnd function was used to compute optimal airspeeds: saving of computation time will occur if this is replaced with either a pre-computed lookup table of best speeds-to-fly and energy change for representative values of horizontal and vertical components of wind or an approximator such as a neural network.

\section{Conclusion}

$\mathrm{T}_{\mathrm{an}}^{\mathrm{H}}$ (n) HIS PAPER HAS introduced a method for minimum energy path planning in complex wind fields using an energy map. This energy map is generated by computing the speed to fly which minimizes the total energy lost between way points (or equivalently maximizing the energy gained). The energy-optimal path is computed using wavefront expansion from the goal, keeping track of the cumulative minimum total energy required to reach the goal.

This energy map can immediately indicate whether a feasible path to the goal exists for a particular starting way point and initial total energy. The energy map can also be used as part of a cost function which may include other considerations (e.g. time to reach the goal), and can be used with generic graph-based path planners (for example $A^{*}$ ).

The energy map is computed using a constraint that all transitions must be towards the goal. This constraint means that lower energy paths which first proceed away from the goal may exist, thus the energy map defines an upper bound on the minimum energy required to reach the goal.

\section{References}

\footnotetext{
1 "MM5 Community Model Homepage," .

${ }^{2}$ Langelaan, J. W., "Tree-Based Trajectory Planning to Exploit Atmospheric Energy," Proceedings of the American Control Conference, Seattle, Washington, June 2008.

${ }^{3}$ Langelaan, J. W., "Long Distance/Duration Trajectories for Small UAVs," AIAA Guidance, Navigation and Control Conference, American Institute of Aeronautics and Astronautics, 2007.

${ }^{4}$ MacCready Jr., P. B., "Optimum Airspeed Selector,” Soaring, January-February 1958, pp. 10-11.

${ }^{5}$ Cochrane, J. H., "MacCready Theory with Uncertain Lift and Limited Altitude," Technical Soaring, Vol. 23, No. 3, July 1999, pp. 88-96.

${ }^{6}$ Reichmann, H., Cross-Country Soaring, Thomson Publications, Santa Monica, California, 1978.

${ }^{7}$ Arho, R., "Optimal Dolphin Soaring as a Variational Problem," OSTIV Publication XIII, Organisation Scientifique et Technique Internationale du Vol à Voile, 1974.

${ }^{8}$ Metzger, D. E. and Hedrick, J. K., "Optimal Flight Paths for Soaring Flight," Journal of Aircraft, Vol. 12, No. 11, 1975, pp. $867-871$.
} 
${ }^{9}$ Sandauer, J., "Some Problems of the Dolphin-Mode Flight Technique," OSTIV Publication XV, Organisation Scientifique et Technique Internationale du Vol à Voile, 1978.

${ }^{10}$ de Jong, J. L., "The Convex Combination Approach: A Geometric Approach to the Optimization of Sailplane Trajectories," OSTIV Publication XVI, Organisation Scientifique et Technique Internationale du Vol à Voile, 1981, pp. 182-201.

${ }^{11}$ Pierson, B. L. and Chen, I., "Minimum Altitude Loss Soaring in a Specified Vertical Wind Distribution," NASA Conference Publication 2085, Science and Technology of Low Speed and Motorless Flight, edited by P. W. Hanson, NASA, Hampton, Virginia, March 1979, pp. 305-318.

${ }^{12}$ Sander, G. and Litt, F. X., "On Global Optimal Sailplane Flight Strategy," NASA Conference Publication 2085, Science and Technology of Low Speed and Motorless Flight, edited by P. W. Hanson, NASA, Hampton, Virginia, March 1979, pp. $355-376$.

${ }^{13}$ Allen, M. J., "Autonomous Soaring for Improved Endurance of a Small Uninhabited Air Vehicle," 43rd AIAA Aerospace Sciences Meeting and Exhibit, American Institute of Aeronautics and Astronautics, Reno, Nevada, January 2005.

${ }^{14}$ Allen, M. J. and Lin, V., "Guidance and Control of an Autonomous Soaring Vehicle with Flight Test Results," AIAA Aerospace Sciences Meeting and Exhibit, AIAA Paper 2007-867, American Institute of Aeronautics and Astronautics, Reno, Nevada, January 2007.

${ }^{15}$ Edwards, D. J., "Implementation Details and Flight Test Results of an Autonomous Soaring Controller," AIAA Guidance, Navigation and Control Conference, American Institute of Aeronautics and Astronautics, Reston, Virginia, August 2008.

${ }^{16}$ Torroella, J. C. R., Long Range Evolution-based Path Planning for UAVs through Realistic Weather Environments, Master's thesis, University of Washington, Seattle, Washington, 2004.

${ }^{17}$ Jardin, M. R. and Bryson, A. E., "Neighboring Optimal Aircraft Guidance in Winds," Journal of Guidance, Control and Dynamics, Vol. 24, No. 4, 2001, pp. 710-715.

${ }^{18}$ Stentz, A., "The Focussed D* Algorithm for Real-Time Replanning," Proceedings of the International Joint Conference on Artificial Intelligence, August 1995.

${ }^{19}$ Anisi, D. A., Robinson, J. W. C., and Ogren, P., "On-line Trajectory Planning for Aerial Vehicles: a Safe Approach with Guaranteed Task Completion," AIAA Guidance, Navigation and Control Conference, AIAA Paper 2006-6107, American Institute of Aeronautics and Astronautics, Keystone, Colorado, August 2006.

${ }^{20}$ Cormen, T. H., Leiserson, C. E., Rivest, R. L., and Stein, C., Introduction to Algorithms, MIT Press, Cambridge, Massachusetts, second edition ed., 2001.

\section{Appendix: Vehicle Properties}

Simulation results are based on the $\mathrm{RnR}$ products $\mathrm{SB}-\mathrm{XC}$ radio control glider.

Note that a fourth order polynomial is used to relate $C_{D}$ to $C_{L}$ : this provided a better fit to the computed data over the full speed range. The speed polar is shown in Figure 9.

Table 1. Parameters for SB-XC glider.

\begin{tabular}{ccc}
\hline \hline variable & value & description \\
\hline $\mathrm{m}$ & $10 \mathrm{~kg}$ & mass \\
$\mathrm{S}$ & $1 \mathrm{~m}^{2}$ & wing area \\
$f\left(C_{L}\right)$ & $0.1723 C_{L}^{4}-0.3161 C_{L}^{3}+0.2397 C_{L}^{2}$ & \\
& $-0.0624 C_{L}+0.0194$ & \\
$v_{a, \min }$ & $12 \mathrm{~m} / \mathrm{s}$ & \\
$v_{a, \max }$ & $35 \mathrm{~m} / \mathrm{s}$ & \\
\hline \hline
\end{tabular}

12 of 13 


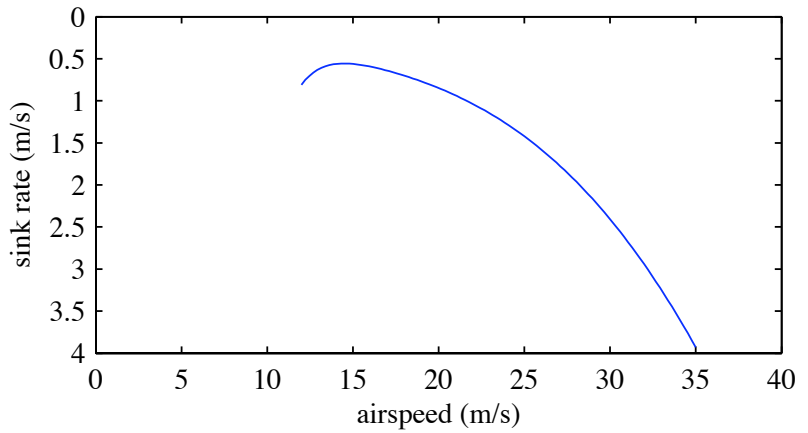

Figure 9. Sink rate vs. airspeed for the SB-XC. Minimum sink is approximately $0.56 \mathrm{~m} / \mathrm{s}$ and occurs at approximately $14.6 \mathrm{~m} / \mathrm{s}$. 